\title{
Evaluation of Polycystic Ovary Syndrome Patients with Strain Echocardiography
}

\author{
Gamze Aslan ${ }^{*}$, Recep Cagdas Aslan², Leyla Elif Sade ${ }^{1}$, Ugur Bal', Gogsen Onalan², Hulusi Bulent Zeyneloglu², \\ Esra Kuscu $^{2}$, Haldun Muderrisoglu ${ }^{1}$
}

\begin{abstract}
Objectives: Polycystic Ovary Syndrome (PCOS) is characterized by several metabolic abnormalities that may lead to insulin resistance, diabetes, and atherosclerosis which are associated with chronic inflammatory processes and oxidative stress. Due to this fact PCOS patients are at increased risk of cardiovascular diseases. We used echocardiographic quantification tools to detect subclinical changes in myocardial functions.

Materials and Methods: Echocardiographic, hormonal and metabolic measurements were performed in twenty-six women with PCOS and twenty-three healthy volunteers. The age of the attendants ranged between 20 and 31 years. PCOS was diagnosed by using the Rotterdam criteria. We compared the myocardial functions of PCOS patients without any cardiovascular symptoms with healthy volunteers by using strain echocardiography.

Results: No differences were found between the two groups' strain, strain rate and myocardial velocity measurements.

Conclusion: According to our study PCOS patients without any clinical cardiovascular symptoms have no impairment in myocardial functions. These results should be further confirmed in larger controlled studies.

Keywords: Polycystic Ovary Syndrome, Echocardiography, Myocardium
\end{abstract}

\section{Introduction}

Polycystic Ovary Syndrome (PCOS) is a disorder of the reproductive system and is seen in 5 to $10 \%$ of the women in reproductive ages (1). Its main features are hyperandrogenism both clinical or biochemical and/or chronic oligo-anovulation and/or ultrasonographic appearance of the polycystic ovaries (2).

There are many studies showing the relationship between PCOS and Cardiovascular Diseases (CVD). Insulin resistance, obesity, hypertension, hyperlipidemia, hypercoagulability, and endothelial dysfunction can be seen frequently in PCOS patients along with increased cardiovascular risks (3). Previously published data show that coronary atherosclerosis and increased carotid intima-media thickness can be observed in PCOS (4).

The aim of our study was to determine the effects of metabolic factors on myocardial functions in PCOS patients, by measuring the strain (S) and strain rate (SR) as means of quantification of myocardial functions, which were first described by Heimdal et al. to define the global and regional myocardial dysfunction (5), and to identify the possible subclinical impairment of left ventricular myocardial function because of the metabolic components of PCOS.

\section{Materials and Methods \\ Study population}

This study was carried out with 26 PCOS patients referred to the Obstetrics and Gynecology Clinic of Baskent University Hospital between December 2008 and September 2009 and 23 healthy female volunteers as control group. The PCOS patients were diagnosed in accordance with 2003 Rotterdam criteria (2). There were neither menstrual cycle abnormalities nor hyperandrogenism in the control group both clinically and biochemically. Clinical symptoms of hyperandrogenism were acne and/or hirsutism. A score higher than 7 based on the modified Ferriman-Gallwey Scale was noted as hirsutism. Any disease other than PCOS including hypertension, diabetes, thyroid function abnormalities, Cushing syndrome, asthma, and other auto-immune diseases, and all medical conditions which caused regular drug use were exclusion criteria for our study. All participants signed the informed consent before enrolling in our study.

A physical examination with a detailed anamnesis including body weight and height were performed in all participants. Body Mass Index (BMI) was determined as body weight (kilograms) divided by height (meters) squared. 


\section{Laboratory measurements}

The participants having a regular menstrual cycle gave their blood samples between third and seventh day of their cycle. The participants with irregular menstrual cycle gave their blood samples irrespective of their cycle day. The blood samples of the participants were taken between 08:00-12:00 am, after 12 hours of fasting.

High sensitive C-reactive protein (hsCRP) was measured with a turbidimetric method by using an Abbott ${ }^{\circledR}$ Architect C8000 device. Total cholesterol, triglyceride, Low Density Lipoprotein (LDL), and High Density Lipoprotein (HDL) were measured with an enzymatic colorimetric method by using a Roche/Hitachi Modular PP (Roche Diagnostics $\mathrm{GmbH}$, Mannheim, Germany). Serum glucose levels were detected with hexokinase method by using a Roche/Hitachi Modular PP (Roche Diagnostics GmbH, Mannheim, Germany). Hemogram tests were performed after the anticoagulation with K3EDTA by using an Abbott Cell-Dyne ${ }^{\circledR} 3700$ System (Abbott Diagnostics, Santa Clara, CA, USA).

Estradiol (e2), Follicle Stimulating Hormone (FSH), Luteinizing Hormone ( $\mathrm{LH})$, prolactin, and insulin levels were measured with an immunometric method by using an Immulite $^{\circledR} 2000$ (Siemens Medical Solutions Diagnostics, Los Angeles, CA). Free testosterone tests were performed with Radioimmunoassay method (RIA) (Diagnostics Systems Laboratories, Webster, TX). 17-OH-progesterone tests were performed with RIA by using BioSource Europe S.A., Nivelles, Belgium.

The Homeostasis Model Assessment Insulin Resistance index (HOMA-IR) was determined by using the following formula: fasting insulin $(\mathrm{mU} / \mathrm{L}) \times$ fasting glucose $(\mathrm{mg} /$ dL)/22.5 (6). HOMA-IR value more than 2.5 was indicative of insulin resistance (7).

\section{Echocardiographic examination}

Transthoracic echocardiographic examinations were conducted with a commercially available cardiac ultrasound system (Acuson Sequoia C256, Acuson Siemens, Mountain View, CA, USA) equipped with a broadband $3.5 \mathrm{MHz}$ transducer with second harmonic capabilities. All patients were examined with standard 2D and Doppler echocardiography conforming to the American Society of Echocardiography (ASE) and European Association of Echocardiography recommendations (8). In addition, longitudinal myocardial systolic and diastolic velocities at the mitral annular level from the lateral and septal sites were measured by using pulsed wave tissue Doppler in the apical 4-chamber view.

Left ventricular longitudinal myocardial systolic and diastolic velocities, systolic SR and systolic $S$ were measured from the apical 2- and 4-chamber views and averaged by using the Syngo WST 3.0 software. A narrow angle with an optimal image depth was used to obtain a high frame rate ( $>100$ frames/s) and avoid angulations. All echocardiographic measurements were performed by the same echocardiographer blinded to the clinical information of the patients.

\section{Statistical analyses}

All analyses were conducted by using the SPSS software (Statistical Package for the Social Sciences, version 15.0, SSPS Inc., Chicago, IL, USA). Continuous variables were expressed as mean \pm Standard Deviation (SD) or median (interquartile range) and categorical variables as percentages. The normality of distribution was tested by the Shapiro-Wilk test. Variables with a normal distribution were compared by a Student-T test or univariate analysis of variance (ANOVA). Variables that showed a non-homogeneous distribution were compared by the Mann Whitney U-test. The comparisons of the categorical variables were performed with the chi-square test. Correlations were evaluated by the Spearman's correlation test. A 'P' value lower than 0.05 was noted as statistically significant.

\section{Results}

Age intervals for PCOS and control group were similar (Table 1). LH, LH/FSH ratio, BMI, fasting serum glucose, HOMA-IR and insulin were higher in PCOS group $(\mathrm{P}<0.05)$ (Table 1). FSH, HDL were lower in PCOS group $(\mathrm{P}<0.05)$ (Table 1$)$. Eight PCOS patients $(31 \%)$ had insulin resistance. There were 10 (39\%) people in PCOS group and $1(4 \%)$ people in the control group in whom BMI values were greater than 30 . The number of people having a BMI between 25 and 29.9 was 7 (27\%) in PCOS group and $5(22 \%)$ in control group. The other participants' BMI values were in normal range. Also, all participants' electrocardiograms were in normal range. Waist circumference measurement and the ratio of waist circumference to thigh circumference were higher in PCOS group $(\mathrm{P}<0.05)$ (Table 1$)$.

No differences were detected between the two groups' conventional echocardiographic measurements (Table 2). Myocardial velocities, strain and strain rate measurements (Table 3) were also similar between the groups both for regional and global indices. No differences were found between the strain measurements of participants whose BMI was greater or less than 25. Similarly, comparing the overweight PCOS patients' (BMI>25) strain measurements with that of normal weight PCOS patients' $(\mathrm{BMI}<25)$ measurements, we found no differences.

\section{Discussion}

PCOS is thought to be an endocrine disorder which increases the cardiovascular risks (9). It was shown that metabolic complications associated with PCOS such as hyperinsulinemia, insulin resistance and dyslipidemia are major risk factors for atherosclerosis (9). In our study, we found fasting glucose (10), insulin, HOMA-IR and BMI to be higher and HDL to be lower in PCOS group $(11,12)$. In PCOS group, obesity and irregularity of sex hormones were more evident as expected. 
Aslan et al.

Table 1. Baseline characteristics and laboratory results of the study population

\begin{tabular}{|c|c|c|c|}
\hline Clinical characteristics & $\mathrm{PCOS}(\mathrm{N}=\mathbf{2 6})$ & Control $(\mathrm{N}=\mathbf{2 3})$ & $\mathbf{P}$ \\
\hline Age (years) & $25.9 \pm 5.8$ & $25.7 \pm 2.8$ & $\mathrm{NS}^{\mathrm{n}}$ \\
\hline Females & $100(\%)$ & $100(\%)$ & $\mathrm{NS}^{\#}$ \\
\hline Body mass index $\left(\mathrm{kg} / \mathrm{m}^{2}\right)$ & $28 \pm 5.1$ & $22 \pm 2.9$ & $0.00^{n}$ \\
\hline Waist circumference $(\mathrm{cm})$ & $84 \pm 12$ & $72 \pm 8.6$ & $0.00^{n}$ \\
\hline Waist to hip ratio & $0.76 \pm 0.06$ & $0.72 \pm 0.05$ & $0.009^{n}$ \\
\hline Hirsutismus & $76.9(\%)$ & $13.0(\%)$ & $0.00^{\#}$ \\
\hline \multicolumn{4}{|l|}{ Laboratory results } \\
\hline Fasting serum glucose (mg/dL) & $90 \pm 8.0$ & $79 \pm 7.0$ & $0.00^{n}$ \\
\hline Total cholesterol level (mg/dL) & $174 \pm 37$ & $167 \pm 30$ & $\mathrm{NS}^{\mathrm{n}}$ \\
\hline $\mathrm{HDL}(\mathrm{mg} / \mathrm{dL})$ & $46 \pm 9$ & $58 \pm 16$ & $0.03^{n}$ \\
\hline $\mathrm{LDL}(\mathrm{mg} / \mathrm{dl})$ & $98 \pm 32$ & $86 \pm 25$ & $N S^{n}$ \\
\hline Triglycerides (mg/dL) & $96 \pm 46$ & $78 \pm 40$ & $\mathrm{NS}^{\mathrm{n}}$ \\
\hline High sensitive C-reactive protein (mg/L) & $2.9 \pm 2.8$ & $1.2 \pm 1.0$ & $\mathrm{NS}^{\mathrm{n}}$ \\
\hline $\mathrm{FSH}(\mathrm{mlU} / \mathrm{ml})$ & $4.9(0.7-7)$ & $6.5(0.8-10)$ & $0.007^{*}$ \\
\hline $\mathrm{LH}(\mathrm{mlU} / \mathrm{ml})$ & $6.3(0.8-21)$ & $4.3(0.7-12)$ & $0.01^{*}$ \\
\hline $\mathrm{LH} / \mathrm{FSH}$ & $1.3(0.3-5)$ & $0.7(0.09-3)$ & $0.00^{*}$ \\
\hline Prolactin (ng/ml) & $12(5-65)$ & $20(5-44)$ & NS* \\
\hline Free testosterone $(\mathrm{pg} / \mathrm{ml})$ & $1.1(0.2-3.1)$ & $0.7(0.3-3.1)$ & NS* \\
\hline DHEA-S $(\mu \mathrm{g} / \mathrm{dl})$ & $240(73-522)$ & $270(114-723)$ & NS* \\
\hline Estradiol (pg/ml) & $44(20-158)$ & $42(20-134)$ & NS* \\
\hline 17-OH-progesteron $(\mu \mathrm{gr} / \mathrm{ml})$ & $1.0(0.4-6.8)$ & $1.2(0.6-2.4)$ & NS* \\
\hline Insulin $(\mu \mathrm{IU} / \mathrm{ml})$ & $5.8(2.8-50)$ & $4.7(2-10)$ & $0.01^{*}$ \\
\hline HOMA-IR value & $2.5 \pm 2.6$ & $0.9 \pm 0.4$ & $0.006^{n}$ \\
\hline Hemoglobin $(\mathrm{g} / \mathrm{dL})$ & $13 \pm 1$ & $13 \pm 1$ & $N S^{n}$ \\
\hline Leucocytes $(1000 / \mu \mathrm{L})$ & $6.303 \pm 2$ & $6.103 \pm 1$ & $N S^{n}$ \\
\hline Thrombocytes $(1000 / \mu \mathrm{l})$ & $290 \pm 73$ & $274 \pm 67$ & $\mathrm{NS}^{\mathrm{n}}$ \\
\hline
\end{tabular}

The median (interquartile range), or frequency counts (percentages), as appropriate. DHEA-S= Dehydroepiandrosterone sulfate; FSH= Follicular Stimulating Hormone; HDL= High Density Lipoprotein; LDL= Low Density Lipoprotein; LH= Luteinizing Hormone; NS= no significant difference; PCOS= Polycystic Ovary Syndrome;

nt-test; *Mann-Whitney $U$-test; " $\chi^{2}$-test.

Our echocardiographic parameters were similar for both groups. The first parameter of our echocardiographic examination was left ventricular ejection fraction and our results were similar for the PCOS patients and the control group, consistent with results of Tekin's study (13). However, Orio et al. found lower left ventricular ejection fraction in overweight PCOS patients than the overweight control group (12). Although $65 \%$ of the PCOS patients and $26 \%$ of the control group in our study were overweight or obese, we could not find similar results to Orio's study for left ventricular ejection fraction measurements. Previous studies suggested that some factors such as obesity, insulin resistance and age could affect the myocardial strain mea- surements. Obesity can cause a subclinical impairment in myocardial functions alone $(14,15)$. Insulin resistant people can show subclinic deterioration in myocardial functions (16). In our study, only $31 \%$ of PCOS patients had insulin resistance and this low percentage of insulin resistant patients can possibly explain the similarity between the PCOS and control groups strain measurements.

Our mitral inflow and mitral velocity results used for measuring left ventricular diastolic functions revealed no differences between both groups as in Tekin's study (13), yet in Erdogan et al. study mitral velocities were lower in PCOS patients (17).

In another study, myocardial peak systolic strain, peak 
Aslan et al.

Table 2. Echocardiographic data of the patients with PCOS and controls

\begin{tabular}{|c|c|c|c|}
\hline Echocardiographic data & $P \cos (\mathrm{N}=26)$ & Control $(\mathrm{N}=23)$ & $\mathbf{P}$ \\
\hline \multicolumn{4}{|l|}{2 Dimensional measurements } \\
\hline IVS (cm) & $0.8(0.5-1.1)$ & $0.7(0.5-1.0)$ & NS* \\
\hline $\mathrm{PW}(\mathrm{cm})$ & $0.8(0.5-1.1)$ & $0.7(0.5-1.0)$ & NS* \\
\hline $\operatorname{LVDD}(\mathrm{cm})$ & $3.9 \pm 0.2$ & $3.7 \pm 0.2$ & $N S^{n}$ \\
\hline $\operatorname{LVSD}(\mathrm{cm})$ & $2.4 \pm 0.2$ & $2.4 \pm 0.2$ & $N S^{n}$ \\
\hline $\mathrm{EF}(\%)$ & $57(51-70)$ & $57(51-66)$ & NS* \\
\hline EDV (ml) & $83(56-124)$ & 79 (57-119) & NS* \\
\hline ESV (ml) & $36 \pm 9$ & $34 \pm 8$ & $N S^{n}$ \\
\hline $\mathrm{SV}(\mathrm{ml})$ & $45 \pm 12$ & $43 \pm 10$ & $N S^{n}$ \\
\hline LVMI $\left(\mathrm{g} / \mathrm{m}^{2}\right)$ & $55 \pm 14$ & $48 \pm 9$ & $N S^{n}$ \\
\hline \multicolumn{4}{|l|}{ Tissue Doppler measurements } \\
\hline Mitral lateral S velocity $(\mathrm{cm} / \mathrm{s})$ & $11 \pm 2$ & $11 \pm 3$ & $N S^{n}$ \\
\hline Mitral septal S velocity $(\mathrm{cm} / \mathrm{s})$ & $8.3 \pm 1.8$ & $8.7 \pm 2.8$ & $N S^{n}$ \\
\hline Mitral lateral E' velocity (cm/s) & $17 \pm 3$ & $17 \pm 4$ & $N S^{n}$ \\
\hline Mitral septal E' velocity $(\mathrm{cm} / \mathrm{s})$ & $13 \pm 2$ & $14 \pm 4$ & $N S^{n}$ \\
\hline Mitral E-wave (cm/s) & $77 \pm 25$ & $81 \pm 12$ & $N S^{n}$ \\
\hline Mitral A-wave (cm/s) & $56 \pm 9$ & $61 \pm 10$ & $N S^{n}$ \\
\hline$E / A$ & $1.4 \pm 0.5$ & $1.3 \pm 0.2$ & $N S^{n}$ \\
\hline E/E' lateral & $4.4 \pm 1.5$ & $5.0 \pm 1.3$ & $N S^{n}$ \\
\hline E/E' septal & $6.1 \pm 2.1$ & $5.9 \pm 1.8$ & $N S^{n}$ \\
\hline \multicolumn{4}{|c|}{ Measurements related to doppler assessment } \\
\hline Baseline heart rate (beats/min) & $69 \pm 11$ & $71 \pm 18$ & $N S^{n}$ \\
\hline Baseline SBP (mmHg) & $114 \pm 9$ & $114 \pm 8$ & $N S^{n}$ \\
\hline Baseline DBP (mmHg) & $74 \pm 7$ & $74 \pm 7$ & $N S^{n}$ \\
\hline
\end{tabular}

A, late mitral inflow velocity; DBP, Diastolic Blood Pressure; E, early mitral inflow velocity; E', early mitral annular velocity; EDV, End Diastolic Volume; EF, Ejection Fraction; ESV, End Systolic Volume; IVS, Interventricular Septum; LVDD, Left Ventricular Diastolic Diameter; LVMI, Left Ventricular Mass Index; LVSD, Left Ventricular Systolic Diameter; PCOS, Polycystic Ovary Syndrome; PW, Posterior Wall; S, Systolic; SBP, Systolic Blood Pressure; SV, Stroke Volume.

n t-test; *Mann-Whitney U-test.

early diastolic strain, and peak early diastolic velocity measurements were found to be lower in obese (BMI>30), insulin resistant PCOS patients (18). However, we could not find any difference in our PCOS group. This can be explained by the small number of insulin resistant PCOS patients $[8(31 \%)]$ in our study.

In a study of overweight PCOS patients, longitudinal strain measurements were lower than the control group (17). The same study found higher HOMA-IR and insulin levels in PCOS group than the control group, yet the percentage of insulin resistant participants was not mentioned (17). Although the PCOS patients' insulin, HOMA-IR and BMI values were higher than the control group in our study, we did not find any impairment of the left ventricular function in both groups. This could be explained by the young age of the participants in our study resulting in shorter durations as PCOS. Cardiological examination should be advised to these patients in the future.

\section{Conclusion}

In conclusion, according to our study PCOS patients with no clinical cardiovascular symptoms have no impairment in myocardial functions. Larger controlled studies are needed for further understanding and validating our results.

\section{Ethical issues}

Approval of the local ethics committee from "Baskent University Clinical Research Ethics Committee" was taken before the study was begun. 
Aslan et al.

Table 3. Regional quantitative myocardial functional data of the patients with PCOS and controls

\begin{tabular}{|c|c|c|c|}
\hline Myocardial functional data & $\mathrm{PCOS}(\mathrm{N}=26)$ & Control ( $\mathrm{N}=23)$ & $\mathbf{P}$ \\
\hline \multicolumn{4}{|l|}{ Systolic Strain measurements } \\
\hline Strain lateral basal (\%) & $21.3 \pm 8.6$ & $19.6 \pm 5.6$ & $N S^{n}$ \\
\hline Strain lateral mid (\%) & $17.9 \pm 5.6$ & $15.6 \pm 5.8$ & $\mathrm{NS}^{\mathrm{n}}$ \\
\hline Strain lateral apical (\%) & $10.7 \pm 4.9$ & $12.1 \pm 7.3$ & $N S^{n}$ \\
\hline Strain septum basal (\%) & $17.2 \pm 6.7$ & $16.3 \pm 9.3$ & $N S^{n}$ \\
\hline Strain septum mid (\%) & $17.9 \pm 5.2$ & $17.4 \pm 5.2$ & $N S^{n}$ \\
\hline Strain septum apical (\%) & $13.9 \pm 6.8$ & $15.6 \pm 6.2$ & $N S^{n}$ \\
\hline Strain anterior basal (\%) & $21.8 \pm 6.8$ & $20.8 \pm 8.4$ & $\mathrm{NS}^{\mathrm{n}}$ \\
\hline Strain anterior mid (\%) & $15.1 \pm 6.3$ & $15.6 \pm 5.1$ & $\mathrm{NS}^{\mathrm{n}}$ \\
\hline Strain anterior apical (\%) & $10 \pm 4.7$ & $12 \pm 6.0$ & $N S^{n}$ \\
\hline Strain inferior basal (\%) & $17.6 \pm 7.8$ & $18.2 \pm 7.5$ & $N S^{n}$ \\
\hline Strain inferior mid (\%) & $16.7 \pm 6.3$ & $18.4 \pm 5.6$ & $N S^{n}$ \\
\hline Strain inferior apical (\%) & $16.6 \pm 7.0$ & $18.7 \pm 6.1$ & $N S^{n}$ \\
\hline \multicolumn{4}{|l|}{ Mean systolic strain } \\
\hline Apical 4 chamber & $16.7 \pm 3.2$ & $16.1 \pm 3.9$ & $N S^{n}$ \\
\hline Two chamber & $16.1 \pm 3.8$ & $17.4 \pm 4.0$ & $N^{n}$ \\
\hline \multicolumn{4}{|l|}{ Systolic Strain Rate, $1 / \mathrm{s}$} \\
\hline Strain rate lateral-basal, $1 / \mathrm{s}$ & $1.2 \pm 0.5$ & $1.1 \pm 0.3$ & $N S^{n}$ \\
\hline Strain rate lateral-mid, $1 / \mathrm{s}$ & $1.0 \pm 0.3$ & $0.9 \pm 0.3$ & $N^{n}$ \\
\hline Strain rate lateral-apical, $1 / \mathrm{s}$ & $0.6 \pm 0.3$ & $0.8 \pm 0.4$ & $N^{n}$ \\
\hline Strain rate septum-basal, $1 / \mathrm{s}$ & $1.0 \pm 0.4$ & $1.0 \pm 0.7$ & $N S^{n}$ \\
\hline Strain rate septum-mid, $1 / \mathrm{s}$ & $1.0 \pm 0.2$ & $0.9 \pm 0.2$ & $N S^{n}$ \\
\hline Strain rate septum-apical, $1 / \mathrm{s}$ & $0.8 \pm 0.3$ & $0.9 \pm 0.2$ & $N S^{n}$ \\
\hline Strain rate anterior-basal, $1 / \mathrm{s}$ & $1.2 \pm 0.4$ & $1.3 \pm 0.6$ & $N S^{n}$ \\
\hline Strain rate anterior-mid, $1 / \mathrm{s}$ & $0.8 \pm 0.3$ & $0.9 \pm 0.3$ & $N S^{n}$ \\
\hline Strain rate anterior-apical, $1 / \mathrm{s}$ & $0.6 \pm 0.2$ & $0.7 \pm 0.3$ & $N^{n}$ \\
\hline Strain rate inferior-basal, $1 / \mathrm{s}$ & $1.0 \pm 0.6$ & $1.1 \pm 0.5$ & $\mathrm{NS}^{\mathrm{n}}$ \\
\hline Strain rate inferior-mid, $1 / \mathrm{s}$ & $0.9 \pm 0.3$ & $1.1 \pm 0.6$ & $N S^{n}$ \\
\hline Strain rate inferior-apical, $1 / \mathrm{s}$ & $0.9 \pm 0.4$ & $1.2 \pm 0.5$ & $N S^{n}$ \\
\hline \multicolumn{4}{|l|}{ Mean systolic strain rate } \\
\hline Apical 4 chamber & $0.9 \pm 0.2$ & $0.9 \pm 0.2$ & $N S^{n}$ \\
\hline Two chamber & $0.9 \pm 0.2$ & $1.1 \pm 0.3$ & $N S^{n}$ \\
\hline \multicolumn{4}{|l|}{ Systolic Velocity (cm/sn) } \\
\hline Velocity lateral-basal, cm/sn & $4.2 \pm 1.5$ & $4.0 \pm 1.1$ & $\mathrm{NS}^{\mathrm{n}}$ \\
\hline Velocity lateral-mid, cm/sn & $2.3 \pm 1.4$ & $2.3 \pm 1.2$ & $N S^{n}$ \\
\hline Velocity lateral-apical, cm/sn & $0.7 \pm 0.7$ & $0.8 \pm 0.6$ & $N S^{n}$ \\
\hline Velocity septum-basal, $\mathrm{cm} / \mathrm{sn}$ & $4.4 \pm 1.1$ & $4.4 \pm 0.7$ & $N S^{n}$ \\
\hline Velocity septum-mid, cm/sn & $2.4 \pm 0.6$ & $2.8 \pm 1.0$ & $N S^{n}$ \\
\hline Velocity septum-apical, cm/sn & $1.0 \pm 0.8$ & $0.9 \pm 0.7$ & $N S^{n}$ \\
\hline Velocity anterior-basal, cm/sn & $3.8 \pm 1.5$ & $4.1 \pm 1.3$ & $N S^{n}$ \\
\hline Velocity anterior-mid, cm/sn & $1.7 \pm 0.7$ & $2.1 \pm 1.2$ & $N^{n}$ \\
\hline Velocity anterior-apical, $\mathrm{cm} / \mathrm{sn}$ & $0.4 \pm 0.3$ & $0.6 \pm 0.5$ & $N S^{n}$ \\
\hline Velocity inferior-basal, $\mathrm{cm} / \mathrm{sn}$ & $4.5 \pm 1.0$ & $4.7 \pm 1.3$ & $N^{n}$ \\
\hline Velocity inferior-mid, $\mathrm{cm} / \mathrm{sn}$ & $2.8 \pm 0.8$ & $3.0 \pm 0.7$ & $N S^{n}$ \\
\hline Velocity inferior-apical, cm/sn & $0.8 \pm 0.4$ & $1.0 \pm 0.4$ & $N S^{n}$ \\
\hline \multicolumn{4}{|l|}{ Mean Systolic Velocity (cm/sn) } \\
\hline Apical 4 chamber & $2.4 \pm 0.6$ & $2.6 \pm 0.5$ & $N S^{n}$ \\
\hline Two chamber & $2.4 \pm 0.6$ & $2.6 \pm 0.6$ & $N S^{n}$ \\
\hline
\end{tabular}

n $t$-test. 


\section{Financial Support}

The study was financially supported by Baskent University (Ankara, Turkey).

\section{Conflict of interests}

We declare that we have no conflict of interests.

\section{Acknowledgments}

We have no acknowledgments to disclose.

\section{References}

1. Franks S. Polycystic ovary syndrome. N Engl J Med 1995;333(13):853-61.

2. Group TREA-sPcw. Revised 2003 consensus on diagnostic criteria and long-term health risks related to polycystic ovary syndrome (PCOS). Hum Reprod 2004;19(1):41-7.

3. Lo JC, Feigenbaum SL, Yang J, Pressman AR, Selby JV, Go AS. Epidemiology and adverse cardiovascular risk profile of diagnosed polycystic ovary syndrome. J Clin Endocrinol Metab 2006;91(4):1357-63.

4. Legro RS. Polycystic ovary syndrome and cardiovascular disease: a premature association? Endocr Rev 2003;24(3):302-12.

5. Heimdal A, Stoylen A, Torp H, Skjaerpe T. Real-time strain rate imaging of the left ventricle by ultrasound. J Am Soc Echocardiogr 1998;11(11):1013-9.

6. Oktem M, Ozcimen EE, Uckuyu A, Esinler I, Pamuk B, Bayraktar N, et al. Polycystic ovary syndrome is associated with elevated plasma soluble CD40 ligand, a marker of coronary artery disease. Fertil Steril 2009;91(6):2545-50.

7. Bonora E, Kiechl S, Willeit J, Oberhollenzer F, Egger G, Targher G, et al. Prevalence of insulin resistance in metabolic disorders: the Bruneck Study. Diabetes 1998;47(10):1643-9.

8. Lang RM, Bierig M, Devereux RB, Flachskampf FA, Foster E, Pellikka PA, et al. Recommendations for chamber quantification: a report from the American Society of Echocardiography's Guidelines and Standards Committeeand the ChamberQuantification Writing Group, developed in conjunction with the European Association of Echocardiography, a branch of the European Society of Cardiology. J Am Soc Echocardiogr 2005;18(12):1440-63.

9. Dokras A. Cardiovascular disease risk factors in polycystic ovary syndrome. Semin Reprod Med 2008;26(1):39-44.

10. Gambineri A, Patton L, Altieri P, Pagotto U, Pizzi C, Manzoli L, et al. Polycystic ovary syndrome is a risk factor for type 2 diabetes: results from a long-term prospective study. Diabetes 2012;61(9):2369-74.

11. Lim SS, Norman RJ, Davies MJ, Moran LJ. The effect of obesity on polycystic ovary syndrome: a systematic review and meta-analysis. Obes Rev 2013;14(2):95109.

12. Orio F Jr, Palomba S, Spinelli L, Cascella T, Tauchmanova L, Zullo F, et al. The cardiovascular risk of young women with polycystic ovary syndrome: an observational, analytical, prospective case-control study. J Clin Endocrinol Metab 2004;89(8):3696-701.

13. Tekin A, Tekin G, Colkesen Y, Kilicdag EB, Bashan I, Sezgin AT, et al. Left ventricular function in patients with polycystic ovary syndrome: a Doppler echocardiographic study. Exp Clin Endocrinol Diabetes 2009;117(4):165-9.

14. Tumuklu MM, Etikan I, Kisacik B, Kayikcioglu M. Effect of obesity on left ventricular structure and myocardial systolic function: assessment by tissue Doppler imaging and strain/strain rate imaging. Echocardiography 2007;24(8):802-9.

15. Orhan AL, Uslu N, Dayi SU, Nurkalem Z, Uzun F, Erer $\mathrm{HB}$, et al. Effects of isolated obesity on left and right ventricular function: a tissue Doppler and strain rate imaging study. Echocardiography 2010;27(3):236-43.

16. Peterson LR, Herrero P, Schechtman KB, Racette $\mathrm{SB}$, Waggoner AD, Kisrieva-Ware $Z$, et al. Effect of obesity and insulin resistance on myocardial substrate metabolism and efficiency in young women. Circulation 2004;109(18):2191-6.

17. Erdogan E, Akkaya M, Bacaksiz A, Tasal A, Turfan M, $\mathrm{Kul} \mathrm{S}$, et al. Subclinical left ventricular dysfunction in women with polycystic ovary syndrome: an observational study. Anadolu Kardiyol Derg 2013;13(8):784-90.

18. Kosmala W, O’Moore-Sullivan TM, Plaksej R, Kuliczkowska-Plaksej J, Przewlocka-Kosmala M, Marwick TH. Subclinical impairment of left ventricular function in young obese women: contributions of polycystic ovary disease and insulin resistance. J Clin Endocrinol Metab 2008;93(10):374854.

Copyright $@ 2014$ The Author(s); This is an open-access article distributed under the terms of the Creative Commons Attribution License (http://creativecommons.org/licenses/by/4.0), which permits unrestricted use, distribution, and reproduction in any medium, provided the original work is properly cited. 